\title{
Evaluating the Grit and Self-Control of U.S. Military College Students through Structural Equation Modeling Methods
}

\author{
Vasiliki Georgoulas-Sherry*
}

Teachers College, Columbia University, New York, United States

*Corresponding author: Georgoulas-Sherry V, Teachers College, Columbia University, 525 West 120th St., New York, NY 10027, United States; Tel: +1 212-678-3000; E-mail: vg2346@tc.columbia.edu

Received: October 14, 2020; Accepted: October 21, 2020; Published: October 28, 2020

\begin{abstract}
Research in positive psychology has revealed the need to examine psychological constructs such as grit and self-control that are vital in shaping predicting and influencing human development and performance. Limited empirical work has assessed the relationships that exist between grit and self-control as they are significant protective mechanisms in promoting positive individual mental health and well-being. Through several structural equation modeling (SEM) techniques, the present study bridges this gap to evaluate the relationship between these two constructs. Recruiting participants $(\mathrm{N}=107)$ from a private U.S. military university ( $=107$ ), military cadet participants completed the Grit Scale and the Brief Self-Control Scale (BSCS). Bi-variate correlations matrixes showed significant relationships between self-control and grit. Confirmatory factor analyses (CFAs) suggested bi-factor models for grit and self-control. Supplementary CFAs revealed a two-factor model structure between grit and self-control. Recommendations for future research are provided.
\end{abstract}

Keywords: Self-control; Grit; Mental health; Positive psychology, Well-being

\section{Introduction}

Recent work in the field of positive psychology has promoted the need to better understand various positive psychology constructs such as grit (e.g., ability to persist over a long period of time regardless of obstacles) and self-control (e.g., capacity to regulate attention, behavior, and emotions despite temptation), including evaluating the association between these constructs, as they are integral in shaping predicting, and influencing human performance [1-4]. Current work has revealed that the need for inhibitory control and perseverance is vital in predicting retention rate and academic success [5,4], dispositional optimism, life satisfaction, and positive affect [6,1], good adjustment and less pathology [7], and health and public safety [8]. Comprehension of the relationship between these two constructs is important in cognitively, mentally, and physically taxing contexts [9]. As our society continues to be exposed to adversity and trauma, there is a value in placing a focus in understanding grit and self-control as these two constructs are crucial protective mechanisms in one's well-being and mental health.

Citation: Georgoulas-Sherry V. Evaluating the Grit and Self-Control of U.S. Military College Students through Structural Equation Modeling Methods. J Anxiety Depress. 2020;3(2):125. 
www.yumedtext.com | October-2020 | ISSN: 2582-3264 | https://dx.doi.org/10.46527/2582-3264.125

Comprehending these positive constructs is incalculable as these negative contexts continue to generate irrevocable physiological and psychological wounds.

Research has evaluated the prevalence of negative life events suggesting that the majority of the U.S. society will be exposed to at least one experience distinct from what is deemed as a typical experience, which can effect an individual's cognitive and mental health, which includes well-being [10,11]. Due to this adverse prevalence, there is justified need to comprehend grit and self-control as these constructs are necessary to support and safeguard individuals from negative environments [10-12]; also, as Armed Forces personnel, and more specifically, trained soldiers, are more likely expected to experience adversity, evaluating these constructs within this context is imperative. Being able to perform vigorously and persistently toward challenges without losing energy or effort over a long period of time regardless of disappointment or failure and regulate behaviors and emotions is essential in surmounting such stressors that are inevitable in life. These constructs help produce a variety of defensive systems that safeguard individuals from adverse and stressful situations and environments (e.g., financial hardships, familial challenges, workplace issues); this allows for improved homeostasis during those difficult times, increased positive well-being in mental health, and reduced maladaptive behaviors $[9,4,11]$. Endeavors like this study is important in the comprehension of grit and self-control.

While research endeavors have assessed self-control and grit, and the relationship that exists between these two constructs, empirical work is still limiting. Additionally, the existing need to better assess the psychological constructs that are valuable and integral following adversity, has produced a heightened need to conceptualize the interplays between grit and self-control. More so, research like this one can assist with the operationalizations of grit and self-control that play an important part in evaluating, predicting, and influencing human performance and in encouraging and promoting healthy and appropriate mental and cognitive wellness $[5,4,12,11]$.

\section{Grit and Self-Control}

Grit and self-control have been key elements in effective psychological health and adjustment [3,11,6,13]. First, grit has been defined as one's ability to perform persistently and enthusiastically toward challenges without a lack of effort or energy over a prolonged period of time despite failure or hardship [2,3,4]. A gritty individual can self-maintain, prevail over barriers to successful complete tasks, and sustain the willpower over a long time, regardless of challenges. Second, self-control is the ability to regulate emotion, attention, and behavior regardless of being tempted or enticed [7]. A self-controlled individual can focus and self-regulate regardless of temptations or desires. Research has shown that greater levels of grit have predicted retention in the workplace, marriage, military, and school [14], prosocial behavior [5], and physical health [8]. Research has shown that greater levels of self-control have predicted decreased PTSD symptoms [13] including positive health behaviors [15], depression [16], and improved psychopathology [7].

Even though grit and self-control have been delineated and defined as a unique construct, they are both often linked to one another [2,4,5]. While grit and self-control have shown to be highly correlated with one another and have been used synonymously, grit and self-control are also very distinct from one another [2,3,5]. Self-control has been defined as the ability to regulate behavior, attention, and emotions regardless of temptation [3,7]. Individuals with self-control can regulate attitudes and behaviors without temptation; however, this regulation and lack of temptation is not necessarily towards a specified pursuit 
www.yumedtext.com | October-2020 | ISSN: 2582-3264 | https://dx.doi.org/10.46527/2582-3264.125

of a goal. Equally, while gritty individuals possess a persistence and passion to persevere over a long period of time, they might lack the regulation from other temptations outside of their specific goal. Furthermore, as Duckworth and Gross [5] affirm, "some paragons of self-control lead undistinguished lives devoid of a focused lifelong passion, and some gritty and exceptionally successful people are famously undisciplined in life domains other than their chosen passion" (320).

Grit and self-control are vital constructs in effective psychological health and adjustment including the successful acclimation and adaption to adverse contexts $[5,17,4,11,7]$. Studies has shown that increased levels of grit have been linked to support mental toughness [18], appreciation for the meaning of life [19], and psychological adjustment and well-being [20]. Lower levels of grit have been associated to hopelessness and suicidality [21], mental distress [19], and lower levels of psychological trauma [17]. Furthermore, studies have evaluated that greater levels of self-control have shown to sustain well-being and life satisfaction [22], healthy behaviors and emotions [23]. Lower levels of self-control have been associated to emotional problems [24], negative affect and stress [25], and mental fatigue [24].

\section{Present Study}

Grit and self-control are essential protective elements in supporting positive mental health and well-being, overcoming failures and hardships regardless of temptation. To the author's knowledge, though, there are minimal studies that have assessed the relationships between these constructs to best assess the distinction of the constructs. For that justification, the primary focus of the present endeavor is to investigate the interrelated, but separate constructs. Through SEM methodologies (e.g., correlation matrixes, confirmatory factor analyses (CFA)), this endeavor aims to examine numerous models that best assess the structure and relationship between grit and self-control. The focus of this endeavor is to evaluate the empirical frameworks in assessing empirical indicators across these constructs. The questions proposed include:

i. What is the theoretical relationship between grit and self-control, and more precisely, what is the factor structures of these constructs? To assess the distinct structure of each construct, different model structures will be evaluated to determine best fit.

ii. How are then, grit and self-control related to each other, and more particularly, are there important covariances between grit and self-control?

\section{Methods}

\subsection{Participants}

A sample of 107 participants (i.e., 34\% females and 66\% males) were recruited from a private military institution which houses all five U.S. military branches (i.e., Army, Navy, Marines, Coast Guard, and Air Force). Participants ranged between the ages of 18 to 22 (i.e., most participants were either 18 (34\%) or 19 (38\%)); most participants were freshmen (40\%) or sophomores (34\%). As Corps of Cadets members, more than half of the participants (54.2\%) self-reported as leader within their cadet team.

\subsection{Materials}

The Grit Scale is a 12-item self-report assessment that measures grit. Scale items include "Setbacks don't discourage me. I don't give up easily" and "I finish whatever I begin." The Grit Scale has shown high internal consistency $(\alpha=.85)$ including for factors of Consistency of Interests $(\alpha=.84)$ and Perseverance of Effort $(\alpha=.78)$ [2]. The Brief Self-Control Scale (BSCS) 
www.yumedtext.com | October-2020 | ISSN: 2582-3264 | https://dx.doi.org/10.46527/2582-3264.125

is a 10-item self-report assessment that measures dispositional self-regulatory behavior in four components: emotions, performance, impulses, and thoughts. Scale items include "I'm good at resisting temptation" and "I get distracted easily." The BSCS showed internal reliability $(\alpha=.85)$ and test-retest reliability $(\alpha=.87)$ [7].

\subsection{Procedure}

Approval for this study came from Teacher's College, Columbia University's Institutional Review Board (IRB). Using a crosssectional design, participants were recruited through word-of-mouth and were offered extra course credit; individuals were not excluded by age, class, gender, or race. Participants received a Qualtrics link to complete wo scales, the Grit Scale and the BSCS.

\section{Results}

\subsection{Data analysis}

A sample size of 108 participants was revealed with acceptable statistical power for medium-sized effects (Cohen's $f=.32$ ) utilizing $\mathrm{G}^{*}$ Power 3.1.9.2 [26]. Participants self-reported as moderately gritty $(\mathrm{M}=3.78, \mathrm{SD}=.47)$ and highly self-controlled $(\mathrm{M}=4.47, \mathrm{SD}=0.48)$. There were no significant differences between gender and grit $(\mathrm{t}(105)=.95, \mathrm{p}=.35, N S)$ or self-control $(\mathrm{t}(105)=.34, \mathrm{p}=.74, N S)$. Additionally, there were no significant differences in age and grit $(\mathrm{F}(4,102)=2.12, \mathrm{p}=.08, N S)$, but significant differences were present in age and self-control $(\mathrm{F}(4,102)=2.51, \mathrm{p}=.047)$ suggesting that individuals who were 19 years old were more likely to report as self-controlled $(M=3.75, S D=0.49)$ than individuals who were $20(M=3.36$, $\mathrm{SD}=0.70)$ or $18(\mathrm{M}=3.65, \mathrm{SD}=0.51)$. Grit and self-control were correlated with one another $(\mathrm{r}=.805, \mathrm{p}<.001)(\mathrm{TABLE}$ $1)$.

TABLE 1. Psychological Constructs Covariance Matrix.

\begin{tabular}{|c|c|c|c|c|c|c|}
\hline & \multicolumn{3}{|c|}{ GRIT } & \multicolumn{2}{c|}{ SELF-CONTROL } \\
\hline & 1 & 2 & 3 & 4 & 5 & 6 \\
\hline 1. Grit & 1 & & & & & \\
\hline 2. Consistency of Interest & $0.80^{* *}$ & 1 & & & & \\
\hline 3. Perseverance of Effort & $0.87^{* *}$ & $0.40^{* *}$ & 1 & & & \\
\hline 4. Self-Control & $0.81^{* *}$ & $0.61^{* *}$ & $0.73^{* *}$ & 1 & & \\
\hline 5. General Self-Discipline & $0.79^{* *}$ & $0.58^{* *}$ & $0.72^{* *}$ & $0.92^{* *}$ & 1 & \\
\hline 6. Impulsivity & $0.72^{* *}$ & $0.55^{* *}$ & $0.65^{* *}$ & $0.94^{* *}$ & $0.73^{* *}$ & 1 \\
\hline & $* *$ Correlation is significant at the 0.01 level (2-tailed) & & \\
\hline
\end{tabular}

To analyze the constructs of grit and self-control, CFA models were employed to assess the factor structures and the associations utilizing single-, bi-, and multiple-factor model (i.e., hierarchical model) structures to examine best fit. CFA 
www.yumedtext.com | October-2020 | ISSN: 2582-3264 | https://dx.doi.org/10.46527/2582-3264.125

models examined the structures that best explain the positive constructs via SPSS' Analysis of Moment Structures (AMOS). Pearson $\mathrm{r}$ correlations were utilized to examine the direction and strength between grit and self-control. Assumptions were satisfactory met for this project (1) multivariate outliers were not present in the findings and (2) multivariate normality was not violated as kurtosis and skewness revealed appropriate measures [27].

A CFA model via maximum likelihood (ML) estimation for grit and self-control was constructed: (1) Model 1 was a unidimensional model with all items loading onto a single latent factor, (2) Model 2 was a multi-factor model (two-factor model for grit or self-control) with no hierarchical structure, and (3) Model 3 was a bi-factor model. Traditional model-fit indices were used: (1) Comparative Fit Index (CFI), (2) Tucker-Lewis Index (TLI), and (3) Root Mean Square of Approximation (RMSEA) (TABLE 2).

TABLE 2. CFA Models' Model Fit Statistics and Indexes Associated.

\begin{tabular}{|c|c|c|c|c|c|}
\hline & $\mathrm{c}^{2}$ & df & TLI & RMSEA & CFI \\
\hline \multicolumn{6}{|l|}{ Grit } \\
\hline $\begin{array}{l}\text { Model } 1 \text { (unidimensional model) } \\
\text { Model } 2 \text { (two-factor model) } \\
\text { Model } 3 \text { (bi-factor model) }\end{array}$ & $\begin{array}{l}104.54 \\
96.01 \\
92.40\end{array}$ & $\begin{array}{l}54 \\
53 \\
52\end{array}$ & $\begin{array}{l}.64 \\
.69 \\
.73\end{array}$ & $\begin{array}{l}.09 \\
.09 \\
.07\end{array}$ & $\begin{array}{l}.70 \\
.75 \\
.73\end{array}$ \\
\hline \multicolumn{6}{|l|}{ Self-Control } \\
\hline $\begin{array}{l}\text { Model } 1 \text { (unidimensional model) } \\
\text { Model } 2 \text { (two-factor model) } \\
\text { Model } 3 \text { (bi-factor model) }\end{array}$ & $\begin{array}{l}139.49 \\
139.32 \\
137.56\end{array}$ & $\begin{array}{l}35 \\
34 \\
33\end{array}$ & $\begin{array}{l}.52 \\
.53 \\
.56\end{array}$ & $\begin{array}{l}.17 \\
.17 \\
.15\end{array}$ & $\begin{array}{l}.65 \\
.65 \\
.62\end{array}$ \\
\hline \multicolumn{6}{|l|}{ Grit \& Self-Control } \\
\hline Model 4 & 54.93 & 1 & .91 & .08 & .92 \\
\hline $\begin{array}{r}\text { Notes: } \mathrm{CFI}=\text { Comparativ } \\
\text { Error of Appro }\end{array}$ & $\begin{array}{l}\text { Tucker } \\
\text { lels were }\end{array}$ & stica & $\begin{array}{l}\text { MSE } \\
\text { ifica }\end{array}$ & $\begin{array}{l}\text { ot Mean } \\
.05)\end{array}$ & \\
\hline
\end{tabular}

\subsection{Grit CFA models}

A unidimensional model of grit was constructed for Model 1, however, findings showed poor fit indices. All twelve items loaded significantly (at the $\mathrm{p}<.001$ level) on expressive flexibility with factor loadings ranging from .42 to .89 . The second measurement model, Model 2, was a two-factor model of grit (which comprised of perseverance of effort (i.e., q2, q3, q5, q7, q8, and q11) and consistency of interest (i.e., q1, q4, q6, q9, q10, and q12)). This model generated poor fit indices; all items significantly loaded on perseverance of effort and consistency of interest with factor loadings ranging from .40 to .90 . The final model of grit, Model 3, examined a bi-factor model of grit, which was comprised of grit as the higher-order factor and perseverance of effort and consistency of interest as the first order factors. Like the previous two models, Model 3 generated poor fit indices even though RMSEA was .07. Findings revealed that the bi-factor model of grit was the best of the three models as it adhered the RMSEA's benchmark for good fit (TABLE 2).

\subsection{Self-control CFA models}

Model 1 examined a unidimensional model of self-control. While all items $(\mathrm{N}=10)$ loaded significantly on self-control with factor loadings ranging from .33 to .80 , results revealed overall poor fit indices. Model 2 assesses a two-factor measurement 
www.yumedtext.com | October-2020 | ISSN: 2582-3264 | https://dx.doi.org/10.46527/2582-3264.125

model of self-control (which comprised of general self-discipline (i.e., q1, q2, q5, q6, and q8) and impulsivity (i.e., q3, q4, q7, q9, and q10)); Model 2 generated poor fit indices, however, all items loaded significantly on the two factors with factor loadings ranging from .34 to .84 . Lastly, a bi-factor model of self-control, Model 3 was examined which consisted of self-control as the higher-order factor and general self-discipline and impulsivity as the first order factors. Like the previous models, findings revealed poor fit indices for Model 3. Model 3 was the better of the three models due to proximity in TLI benchmark for good fit (TABLE 2).

\subsection{Girt and self-control CFA model}

A CFA model was utilized to evaluate the model structures that can appropriately explain the best fit relationship between the constructs of grit and self-control. Model 4 evaluated a two-factor measurement model which comprised of two latent factors: (1) grit and (2) self-control. The latent factor of grit comprised of two indicators (i.e., perseverance of effort and consistency of interest). The latent factor of self-control consisted of two indicators (general self-discipline and impulsivity). Model 4 produced suitable fit indices (TABLE 2). Findings revealed statistically significant factor loadings (at the p <.001 level) and in the anticipated direction on this model; results showed Model 4 was suitable due to meeting RMSEA and CFI benchmarks [28]. The latent factor of grit had factor loadings of .48 (i.e., perseverance of effort) and .32 (i.e., consistency of interest). The latent factor of self-control had factor loadings of .67 (i.e., impulsivity) and .80 (i.e., general self-discipline).

\section{Discussion}

Through the utility of SEM methodology to evaluate the factor structures and the associations between grit and self-control, the focus of this endeavor included examining the related, but distinct, psychological constructs. Numerous CFA models were constructed to compute the model structures that best fit each construct. Findings showed bi-factor models best fit grit and selfcontrol. Additional CFAs were completed to examine the most suitable model structure that can evaluate the appropriate relationship between these two constructs.

According to Griffin et al. [28], the bi-factor model of grit and self-control evaluates the direct effects of these measurement models, showing that each observed variable (i.e., each scale's items) contributed variance to the constructs of grit and selfcontrol, respectively; the observed variables portrayed grit and self-control as seen in the bi-factor model structure, not the subfacets. While previous work has shown that grit and self-control would be best assessed through a two-factor model structure, direct effects within each scale's items contributed grit and self-control. Furthermore, findings revealed a two-factor model structure between grit and self-control; these results show that grit and self-control, while theoretically correlated to one another, are different from one another. Prior to this study, these model structures were not previously wholly empirically evaluated, and several implications can emerge from this work. Most importantly, the two-factor model structure supported the vital need to distinguish these two independent but parallel constructs; findings from this study supported previous work [5]. Regardless of empirical considerations and assumptions, theoretical support like this study is essential to assess the relationships between grit and self-control.

\section{Limitations}

A few limitations impacted the findings of this endeavor. First, the sample recruited for this study may have limited the generalizability of the results given that the participants were a military college population with a large proportion of this 
www.yumedtext.com | October-2020 | ISSN: 2582-3264 | https://dx.doi.org/10.46527/2582-3264.125

sample with high levels of grit or self-control. This limitation could have affected regressions and lowered correlations. Additionally, while this study utilized a military sample, this study employed a college population who were most likely not exposed to combat. Future work in this field could benefit from a larger and broader sample of participants can help support potential for generalizability.

Second, even though this study found significant results, findings revealed decreased levels of reliability; this could limit the application of this study's findings. However, this limitation can be found in prior work that has assessed the empirical model structures and relationships of grit and self-control. Despite this limitation, this endeavor does add to the current positive psychology literature and does work to measure the distinction of each construct.

Third, as this study centered on the empirical structure and relationship of grit and self-control, this endeavor could have aided from a non-correlational or cross-sectional approach. While grit and self-control were highly related to one another, as revealed in these findings, further work should focus on evaluating how grit and self-control are linked once these positive constructs are manipulated and isolated; other methodologies might produce distinct and potentially, more important results that cannot be produced or replicated in correlational studies.

\section{Implications and General Conclusion}

Research investigating the relationships and structures of positive psychology constructs are invaluable as positive psychology continues to a variety of contexts, fields, and disciplines [11]. As these types of constructs are vital in impacting positive and healthy human functioning, in promoting psychological health and adjustment, and in overcoming failures and hardships regardless of temptation [5,17,4,11,7]. The findings of this study revealed a two-factor model structure for grit and self-control which encourages the distinction between these constructs; specifically, these results discourage the synonymous use of these constructs are they are empirically different from one another and using these constructs synonymously can be erroneous and misleading. Studies like this one can continue to show the risk in using these constructs in a parallel fashion.

Through SEM methodologies, this project computed numerous models to best explain the structure and relationship between grit and self-control. By comprehending the empirical framework and theoretical association between these two constructs, the scientific community might be more likely to identify possible vulnerabilities to negativity or adversity and facilitate more opportunities to integrate salient concepts of grit and self-control into applicable contexts such as the disciplines of science, medicine, and mental health. Combining these positive constructs can promote a necessary and significant approach to conceptualize challenge and hardship. This study, like others in positive psychology, works to shift focus from conceptualizing trauma in terms of a negative or "deficit-based" paradigm, and instead, focus on the positive consequences that arise from trauma like the deterrence and prevention of dysfunction in attending and understanding psychopathology [11]. Research like this one can further the understanding of the effects of grit and self-control and its impact on individual well-being and mental health.

As this research endeavor justifies the need to better understand grit and self-control, studies like this one can play a crucial role to promote the construction, development, and facilitation of trainings/ interventions and assessments/ scales that center on these constructs. For example, current validated assessment/ scales might benefit from reevaluation to confirm that each positive construct effectively measures the respective constructs. Even more so, existing trainings/ interventions could also 
www.yumedtext.com | October-2020 | ISSN: 2582-3264 | https://dx.doi.org/10.46527/2582-3264.125

benefit from improved curriculums to be more effective to support health outcomes. Studies like this one which can help distinguish these positive psychology constructs, can be vital in strengthening trainings/ interventions and assessments/ scales which can consequently help diminish negative outcome from adversity.

The completion of this proposed endeavor offers support of grit and self-control, two fundamental positive constructs in evaluating, predicting, and influencing human performance and in encouraging and promoting healthy and appropriate mental and cognitive wellness [5,4,12,11]. As such, this proposed project assessed the relationships that exist structurally and empirically between these two constructs that are significant protective elements in promoting individualized mental health and well-being through utilizing a variety of SEM techniques to examine these frameworks and relationships surrounding grit and self-control.

\section{REFERENCES}

1. Baumeister RF, Vohs KD, Tice DM. The strength model of self-control. Curr Dir Psychol Sci. 2007;16(6):351-5.

2. Duckworth AL, Peterson C, Matthews MD, et al. Grit: perseverance and passion for long-term goals. J Pers Soc Psychol. 2007;92(6):1087-1101.

3. Duckworth AL, Taxer JL, Eskreis-Winkler L, et al. Self-control and academic achievement. Annu Rev Psychol. 2019;70:373-99.

4. Maddi SR, Matthews MD, Kelly DR, et al. The role of hardiness and grit in predicting performance and retention of USMA cadets. Mil Psychol. 2012;24(1):19-28.

5. Duckworth A, Gross JJ. Self-control and grit: Related but separable determinants of success. Curr Dir Psychol Sci. 2014;23(5):319-25.

6. Oriol X, Miranda R, Bazán C, et al. Distinct routes to understand the relationship between dispositional optimism and life satisfaction: Self-Control and grit, positive affect, gratitude, and meaning in life. Front Psychol. 2020;11:907.

7. Tangney JP, Baumeister RF, Boone AL. High self-control predicts good adjustment, less pathology, better grades, and interpersonal success. J Pers. 2004;72(2):271-324.

8. Moffitt TE, Arseneault L, Belsky D, et al. A gradient of childhood self-control predicts health, wealth, and public safety. Proc Natl Acad Sci U S A. 2011;108(7):2693-8.

9. Hannah ST, Campbell DJ, Matthews MD. Advancing a research agenda for leadership in dangerous contexts. Mil Psychol. 2010;22(sup1):S157-89.

10. Mobbs MC, Bonanno GA. Beyond war and PTSD: The crucial role of transition stress in the lives of military veterans. Clin Psychol Rev. 2018;59:137-44.

11. Southwick SM, Bonanno GA, Masten AS, et al. Resilience definitions, theory, and challenges: interdisciplinary perspectives. Eur J Psychotraumatol. 2014;5(1):1-14.

12. Nielsen KS, Gwozdz W, De Ridder D. Unraveling the relationship between trait self-control and subjective wellbeing: the mediating role of four self-control strategies. Front Psychol. 2019;10:706.

13. Walter KH, Gunstad J, Hobfoll SE. Self-control predicts later symptoms of posttraumatic stress disorder. Psychol Trauma. 2010;2(2):97-101.

14. Eskreis-Winkler L, Duckworth AL, Shulman EP, et al. The grit effect: Predicting retention in the military, the workplace, school, and marriage. Front Psychol. 2014;5:36. 
www.yumedtext.com | October-2020 | ISSN: 2582-3264 | https://dx.doi.org/10.46527/2582-3264.125

15. Melanko S, Larkin KT. Retracted Article: Preference for immediate reinforcement over delayed reinforcement: relation between delay discounting and health behavior. J Behav Med. 2015;36(1):34-43.

16. Dombrovski AY, Siegle GJ, Szanto K, et al. The temptation of suicide: striatal gray matter, discounting of delayed rewards, and suicide attempts in late-life depression. Psychol Med. 2012;42(6):1203-15.

17. Harris JL, Murray BJ. Educational grit and psychological trauma. Developments in Business Simulation and Experiential Learning: Proceedings of the Annual ABSEL Conference. 2017;44(1).

18. Gucciardi DF, Peeling P, Ducker KJ, et al. When the going gets tough: Mental toughness and its relationship with behavioural perseverance. J Sci Med Sport. 2016;19(1):81-6.

19. Kleiman EM, Adams LM, Kashdan TB, et al. Gratitude and grit indirectly reduce risk of suicidal ideations by enhancing meaning in life: Evidence for a mediated moderation model. J Res Personal. 2013;47(5):539-46.

20. Vinothkumar M, Prasad N. Moderating role of resilience in the relationship between grit and psychological wellbeing. Int J Psychol Psychiatry. 2016;4(2):10-23.

21. Pennings SM, Law KC, Green BA, et al. The impact of grit on the relationship between hopelessness and suicidality. Int J Cogn Ther. 2015;8(2):130-42.

22. Hofmann W, Luhmann M, Fisher RR, et al. Yes, but are they happy? Effects of trait self-control on affective wellbeing and life satisfaction. J Pers. 2014;82(4):265-77.

23. Daly M, Baumeister RF, Delaney L, et al. Self-control and its relation to emotions and psychobiology: Evidence from a Day Reconstruction Method study. J Behav Med. 2014;37(1):81-93.

24. Inzlicht M, Marcora SM. The central governor model of exercise regulation teaches us precious little about the nature of mental fatigue and self-control failure. Front Psychol. 2016;7:656.

25. Hamama L, Ronen T, Shachar K, et al. Links between stress, positive and negative affect, and life satisfaction among teachers in special education schools. J Happiness Stud. 2013;14(3):731-51.

26. Faul F, Erdfelder E, Buchner A, et al. Statistical power analyses using G*Power 3.1: Tests for correlation and regression analyses. Behav Res Methods. 2009;41(4):1149-60.

27. Finney SJ \& DiStefano C. Non-normal and categorical data in structural equation modeling. In: Hancock GR, Mueller RO, editors. Structural equation modeling: A second course. Greenwich, CT: Information Age Publishing, USA; 2006. 269-314 p.

28. Griffin KW, Botvin GJ, Scheier LM, et al. Personal competence skills, distress, and well-being as determinants of substance use in a predominantly minority urban adolescent sample. Prev Sci. 2002;3(1):23-33. 